\title{
Lateral parietal arteriovenous malformation with feeding artery aneurysm
}

\author{
Ashish H. Shah, MD, ${ }^{1}$ Anthony C. Wang, MD, ${ }^{2}$ and Jacques J. Morcos, MD ${ }^{1}$ \\ 'Department of Neurological Surgery, University of Miami, Florida; and \\ ${ }^{2}$ Department of Neurological Surgery, University of California, Los Angeles, California
}

Superficial arteriovenous malformations (AVMs) with favorable Spetzler-Martin grading are amenable to primary surgical resection. Careful preoperative workup including preoperative angiograms is essential to identify feeding artery aneurysms and deep venous drainage. The authors present a 37-year-old female who presented with a Spetzler-Martin Grade II right parietal superficial AVM with a 5-mm feeding artery aneurysm from the posterior cerebral artery. Given the risk of hemorrhage, the AVM was resected completely without any complications. On subsequent postoperative angiograms, the feeding artery aneurysm diminished in caliber. Feeding artery aneurysms may regress spontaneously after resection of an AVM due to flow-related changes.

The video can be found here: https://youtu.be/PpwODc9il3g.

KEYWORDS AVM; feeding artery aneurysm; arteriovenous malformation; video; surgery 